\title{
Infectious disease in respiratory medicine
}

\author{
Brian D. Kent ${ }^{1,2}$ \\ ${ }^{1}$ Dept of Respiratory Medicine, St James' Hospital, Dublin, Ireland. ${ }^{2}$ School of Medicine, Trinity College Dublin, Dublin, Ireland. \\ Corresponding author: Brian D. Kent (briankent@physicians.ie)
}

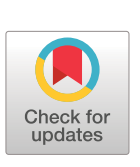

\begin{abstract}
Shareable abstract (@ERSpublications)
COVID-19 has brought respiratory infections into the spotlight, but other infectious diseases remain important causes of respiratory illness and death https://bit.ly/36t6Rhf
\end{abstract}

Cite this article as: Kent BD. Infectious disease in respiratory medicine. Breathe 2022; 18: 220022 [DOI: $10.1183 / 20734735.0022-2022]$.

Copyright OERS 2022

Breathe articles are open access and distributed under the terms of the Creative Commons Attribution Non-Commercial Licence 4.0.

Received: 18 Feb 2022 Accepted: 18 Feb 2022

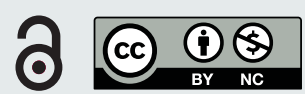

It is over a century since a respiratory disease was at the forefront of the public consciousness to the extent we see today. The emergence of severe acute respiratory syndrome coronavirus 2 (SARS-CoV-2) and the global spread of coronavirus disease 2019 (COVID-19) have devastated lives and crippled healthcare systems around the world.

By necessity, then, the respiratory community’s spotlight has been on COVID-19. But in the shadows, old foes lurk, and other respiratory infections remain important causes of morbidity and mortality.

Perhaps the oldest of these foes is Mycobacterium tuberculosis. Tuberculosis (TB) has been part of the human story since prehistory, with evidence of its baleful influence found everywhere from Egyptian mummies to Mayan temples. In the developed world, it can be easy to dismiss it as a quaint slice of Victoriana, conjuring as it may images of romantic poets wasting away elegantly, but TB remains a global menace, causing in the range of 2 million deaths every year. In this issue of Breathe, GiLL et al. [1] discuss the contemporary management of $\mathrm{TB}$, including the use of emerging therapies and dealing with drug-resistant strains.

The last time a respiratory pathogen caused the levels of global disease and death we have witnessed over the past 2 years was the great influenza pandemic of 1918-1919. Whilst influenza has been largely held in abeyance by the public health measures deployed to tackle COVID-19, we can be confident of its re-emergence as these measures are unwound. In parallel with this, other respiratory viruses, such as respiratory syncytial virus, are likely to make their mark. In a forthcoming article, C. Nunes-Silva and co-authors review non-COVID respiratory viral infections, including their clinical impact and treatment.

During the early stages of the COVID-19 pandemic there was widespread fear that infection with SARS-CoV-2 could be particularly problematic for people living with chronic respiratory disease. Unfortunately, this particularly proved to be the case for those with interstitial lung disease (ILD), with mortality rates approaching 50\% for ILD patients hospitalised due to COVID-19 [2]. This isn't the only relationship between COVID-19 and ILD - a significant minority of patients admitted with COVID-19 pneumonia go on to develop ILD, with associated impacts on lung function and quality of life [3]. These interactions between COVID-19 and ILD are explored in detail by MyALL et al. [4].

The lower airways were historically seen as a largely sterile environment, with any microbes identified within them viewed as threatening interlopers. However, as detailed in a forthcoming article by CAMPBELL et al. [5], the lungs turn out to be home to a rich and diverse microbial flora. As they discuss, whilst the study of the respiratory microbiome is comparatively inchoate, what data we have suggests that it may play an important role in health and disease, and in determining outcomes of treatment for respiratory illness.

It is now over 2 years since the first cases of COVID-19 were reported in Europe. Inevitably, as frontline clinicians delivering care to patients with COVID-19, many colleagues working in respiratory medicine 
across the continent have themselves contracted the virus, with some tragically dying as a result. This issue of Breathe is dedicated to the memories of these "precious friends hid in death's dateless night", and to other respiratory clinicians around the world who have made the ultimate sacrifice in caring for their patients.

Conflict of interest: B.D. Kent has participated in advisory boards and/or received speaker fees from AstraZeneca, Chiesi, GSK, Novartis, and Teva; has received educational travel bursaries from Boehringer Ingelheim, Chiesi, and Napp; and has received research funding from Itamar Medical.

\section{References}

1 Gill CM, Dolan L, Piggott LM, et al. New developments in tuberculosis diagnosis and treatment. Breathe 2022; 18: 210149.

2 Drake TM, Docherty AB, Harrison EM, et al. Outcome of hospitalization for COVID-19 in patients with interstitial lung disease. An international multicenter study. Am J Respir Crit Care Med 2020; 202: 1656-1665.

3 Myall KJ, Mukherjee B, Castanheira AM, et al. Persistent post-COVID-19 interstitial lung disease. An observational study of corticosteroid treatment. Ann Am Thorac Soc 2021; 18: 799-806.

4 Myall KJ, Martinovic JL, West A. How COVID-19 interacts with interstitial lung disease. Breathe 2022; 18: 210158.

5 Campbell CD, Barnett C, Sulaiman I. A clinicians' review of the respiratory microbiome. Breathe 2022; in press [https://doi.org/10.1183/20734735.0161-2022]. 